\title{
Extensions of classical congruences for parameters in binary quadratic forms
}

\author{
by \\ Ronald Evans (La Jolla, CA)
}

1. Introduction. Let $\mathbb{Q}(\sqrt{-k})$ be an imaginary quadratic field with (fundamental) discriminant $-k$ and class number $h$. Define

$$
U=\left\{u \in \mathbb{Z}: 0<u<k \text { and }\left(\frac{-k}{u}\right)=1\right\} \quad \text { and } \quad R=\frac{1}{k} \sum_{u \in U} u .
$$

If $k \notin\{3,4,8\}, R$ is an integer with

$$
R=\phi(k) / 4-h / 2
$$

(see [7, Lemma 2.1]). When $k \notin\{3,4,8\}$, choose integers $t, w$ such that

$$
k=t w, \quad t \text { prime }>2 .
$$

For a prime $p$ with $\left(\frac{-k}{p}\right)=1$, let $r$ denote the smallest positive integer such that

$$
q:=p^{r} \equiv 1(\bmod k) .
$$

Assume that $k \notin\{3,4,8\}$. There are integers $C, D$, unique up to sign, such that $[10]$

$$
4 p^{h}=C^{2}+k D^{2}, \quad p \nmid C .
$$

In accordance with [7, Theorem 3.1], we choose the sign of $C$ so that $C$ is uniquely determined by the congruences (1.2)-(1.4) below. If $w=1$, then

$$
C \equiv 2(-p)^{-R}(\bmod t)
$$

if $w>1$ and $-1 \equiv p^{b}(\bmod w)$ for some positive integer $b$ (taken minimal), then

$$
C \equiv \begin{cases}2 p^{h / 2}(-1)^{R+\phi(k) /(4 b)}(\bmod t) & \text { if } p=2 \\ 2 p^{h / 2}(-1)^{R+\phi(k)\left(w+1+p^{b}\right) /(4 b w)}(\bmod t) & \text { if } p>2\end{cases}
$$

2000 Mathematics Subject Classification: Primary 11E25, 11T24; Secondary 11B65, 11R29, 11S80. 
if -1 is not a power of $p(\bmod w)$, then

$$
C \equiv \begin{cases}2 p^{h / 2}(-1)^{R}(\bmod t) & \text { if } p=2, \\ 2 p^{h / 2}(-1)^{R+\phi(k)(q-1) /(4 k r)}(\bmod t) & \text { if } p>2 .\end{cases}
$$

The significance of the choice of $C$ uniquely determined by (1.1)-(1.4) is that $C$ simultaneously satisfies Stickelberger's [10] classical congruence

$$
C \equiv \prod_{u \in U}[p u / k] !^{-1}(\bmod p)
$$

where $[x]$ denotes the greatest integer $\leq x$. This is proved in $[7]$.

The first object of this paper is to extend (1.5) by determining $C$ $\left(\bmod p^{2}\right)$. More than just a routine application of the Gross-Koblitz formula is needed to accomplish this in a usefully explicit way. Our determination for odd $p$ is formulated in terms of expressions $B_{u}$ (defined in (2.2)) that can be computed $\left(\bmod p^{2}\right)$ for large $p$ relatively rapidly (as indicated near the end of this section). A fast computation of $C$ yields in particular a fast computation of Eisenstein's binomial coefficients $\left(\bmod p^{2}\right)$, in view of Corollaries 2.3 and 4.2 .

The results for $p>2$ are presented in Section 2 (Theorem 2.2). Those for $p=2$ are presented in Section 3 (Theorem 3.1). We remark that in the case $p=2$, Stickelberger's congruence $(1.5)$ is trivial $($ since $C(\bmod 2)$ always equals 1 , by $(1.1))$. In contrast, the determination of $C(\bmod 4)$ is nontrivial, and the results are rather surprising (see Theorem 3.3).

Theorem 2.2 has the following counterparts for the exceptional cases $k \in\{3,4,8\}$. Fix a prime $p$ with $\left(\frac{-k}{p}\right)=1$. For $k=4$, there are integers $a, b$ (with $a$ unique) such that

$$
p=a^{2}+b^{2}, \quad a \equiv 1(\bmod 4)
$$

and

$$
a \equiv\left(2^{p-1}+1\right)^{-1}\left(\begin{array}{l}
{[p / 2]} \\
{[p / 4]}
\end{array}\right)+\frac{p}{2}\left(\begin{array}{l}
{[p / 2]} \\
{[p / 4]}
\end{array}\right)^{-1}\left(\bmod p^{2}\right) .
$$

This is proved in Chowla, Dwork and Evans [3]; for a simpler proof, see $[2$, Theorem 9.4.3]. For $k=3$, there are integers $a, b$ (with $a$ unique) such that

$$
4 p=a^{2}+27 b^{2}, \quad a \equiv-1(\bmod 3)
$$

and

$$
a \equiv\left(\begin{array}{c}
{[2 p / 3]} \\
{[p / 3]}
\end{array}\right)+p\left(\begin{array}{c}
{[2 p / 3]} \\
{[p / 3]}
\end{array}\right)^{-1}\left(\bmod p^{2}\right)
$$


This is proved in [2, Theorem 9.4.2]. Finally, for $k=8$, there are integers $C, D$ (with $C$ unique) such that

$$
4 p=C^{2}+8 D^{2}, \quad C \equiv 2(-1)^{[p / 8]+[p / 2]}(\bmod 8)
$$

and

$$
C \equiv p\left(\begin{array}{l}
{[p / 2]} \\
{[p / 8]}
\end{array}\right)^{-1}+\left(\begin{array}{l}
{[p / 2]} \\
{[p / 8]}
\end{array}\right)\left(2-2^{p-1}-y / 8\right)\left(\bmod p^{2}\right)
$$

where

$$
y=\sqrt{2}(2-\sqrt{2})^{q-1}-\sqrt{2}(2+\sqrt{2})^{q-1} .
$$

This is proved for the case $p \equiv 1(\bmod 8)$ in $[2$, Theorem 9.4.5]. The proof for the remaining case $p \equiv 3(\bmod 8)$ is more complicated and is given in Section 4 (Theorem 4.1). The complications arise because such $p$ does not split into first degree primes in $\mathbb{Q}(\sqrt{-k})$.

Application of the binomial theorem in (1.12) shows that $y \in \mathbb{Z}$, so that $(1.11)$ makes sense even though $\sqrt{2}$ does not exist $(\bmod p)$ when $p \equiv 3$ $(\bmod 8)$. From a computational point of view, it would be too slow to use the binomial expansion to compute the integer $y\left(\bmod p^{2}\right)$ when $p$ is large. It is faster to find $y\left(\bmod p^{2}\right)$ by computing $(2 \pm \sqrt{2})^{q-1}\left(\bmod p^{2}\right)$ via the method of successive squarings. Similar considerations apply to the computation of the integers $B_{u}$ (defined in (2.2)) which appear in Theorem 2.2.

In Corollaries 4.2 and 2.3, we apply Theorems 4.1 and 2.2 to give congruences for Eisenstein's binomial coefficients $\left(\begin{array}{l}{[p / 2]} \\ {[p / 8]}\end{array}\right)$ and $\left(\begin{array}{c}{[3 p / 7]} \\ {[p / 7]}\end{array}\right)\left(\bmod p^{2}\right)$ in the cases $k=8$ and $k=7$, respectively (cf. [2, Section 12.9]). This solves Research Problem \#26 posed in [2, p. 498].

Evaluations of binomial coefficients $\left(\bmod p^{2}\right)$ are of more than just theoretical interest. For example, Crandall, Dilcher and Pomerance [6] have employed such evaluations to speed up computations in the search for Wilson primes.

2. Determination of $C\left(\bmod p^{2}\right)$ when $p>2$. Throughout this section, $p$ is an odd prime with $\left(\frac{-k}{p}\right)=1$. For $\zeta_{k}=\exp (2 \pi i / k)$ and $s \in \mathbb{Z}$, define

$$
A_{s}=\frac{1}{k} \sum_{j=1}^{k-1} \zeta_{k}^{-s j}\left\{\left(1-\zeta_{k}^{j}\right)^{q-1}-1\right\}
$$

and

$$
B_{s}=A_{s}-A_{0}
$$

The following lemma generalizes a result proved for $r=1$ in [2, p. 280]. 
LEMma 2.1. For $1 \leq s \leq k, B_{s}$ is a rational integer multiple of $p$ satisfying

$$
B_{s}=B_{k-s} \equiv \frac{p}{k} \sum_{0<j<p s / k} \frac{1}{j}\left(\bmod p^{2}\right) .
$$

Proof. For any integer $s$, since $q$ is odd,

$$
\begin{aligned}
k\left(A_{s}-A_{s-1}\right) & =\sum_{j=1}^{k-1} \zeta_{k}^{-s j}\left(1-\zeta_{k}^{j}\right)\left(\left(1-\zeta_{k}^{j}\right)^{q-1}-1\right) \\
& =\sum_{j=0}^{k-1} \zeta_{k}^{-s j}\left(\left(1-\zeta_{k}^{j}\right)^{q}-\left(1-\zeta_{k}^{j}\right)\right) \\
& =\sum_{j=0}^{k-1} \zeta_{k}^{-s j} \sum_{m=1}^{q-1}\left(\begin{array}{c}
q \\
m
\end{array}\right)\left(-\zeta_{k}^{j}\right)^{m} \\
& =\sum_{m=1}^{q-1}\left(\begin{array}{c}
q \\
m
\end{array}\right)(-1)^{m} \sum_{j=0}^{k-1} \zeta_{k}^{j(m-s)} .
\end{aligned}
$$

Therefore,

$$
A_{s}-A_{s-1}=\sum_{\substack{0<m<q \\
m \equiv s(\bmod k)}}(-1)^{m}\left(\begin{array}{c}
q \\
m
\end{array}\right) \text {. }
$$

Thus for $s \geq 0$,

$$
B_{s}=\sum_{\nu=1}^{s}\left(A_{\nu}-A_{\nu-1}\right) \in \mathbb{Z}
$$

Since

$$
\left(1-\zeta_{k}^{j}\right)^{q-1}=\left(\zeta_{k}^{-j}-1\right)^{q-1}=\left(1-\zeta_{k}^{-j}\right)^{q-1},
$$

we see from (2.1) that $A_{k-s}=\bar{A}_{s}$, so that by $(2.2)$,

$$
B_{k-s}=A_{k-s}-A_{0}=\bar{A}_{s}-\bar{A}_{0}=\bar{B}_{s}=B_{s} .
$$

It remains to prove the congruence in $(2.3)$. Since $\left(\begin{array}{c}q \\ m\end{array}\right)=(q / m)\left(\begin{array}{c}q-1 \\ m-1\end{array}\right)$, it follows from (2.4) that

$$
A_{s}-A_{s-1} \equiv \sum_{\substack{0<m<q \\
m \equiv s(\bmod k) \\
p^{r-1} \| m}}(-1)^{m}\left(\begin{array}{c}
q \\
m
\end{array}\right)\left(\bmod p^{2}\right) .
$$

Since

$$
\left(\begin{array}{c}
q-1 \\
m-1
\end{array}\right)=\frac{(q-1) \ldots(q-(m-1))}{1 \ldots(m-1)} \equiv(-1)^{m-1}(\bmod p)
$$


we have, writing $m=p^{r-1} n$,

$$
A_{s}-A_{s-1} \equiv-p \sum_{\substack{0<n<p \\ n \equiv s p(\bmod k)}} \frac{1}{n}\left(\bmod p^{2}\right) .
$$

Thus

$$
\begin{aligned}
A_{s}-A_{s-1} & \equiv-p \sum_{\substack{0<n<p \\
n \equiv s p(\bmod k)}} \frac{1}{n-s p}=-p \sum_{\substack{-s p<i<(1-s) p \\
k \mid i}} \frac{1}{i} \\
& =\frac{p}{k} \sum_{(s-1) p / k<j<s p / k} \frac{1}{j}\left(\bmod p^{2}\right),
\end{aligned}
$$

where we have written $n-s p=i=-k j$. The congruence in (2.3) with $1 \leq s \leq k$ now follows from (2.5) and (2.6).

The following theorem generalizes (1.5).

TheOREM 2.2. If $k \notin\{3,4,8\}$ and $p>2$, then

$$
\begin{aligned}
C \equiv & p^{h} \prod_{u \in U}[p u / k] ! \\
& +(-(p-1) !)^{R} \prod_{u \in U}[p u / k] !^{-1}\left(1+\sum_{u \in U} u B_{u}\right)\left(\bmod p^{2}\right) .
\end{aligned}
$$

Proof. In $[7,(2.28)]$, we wrote $(C+D \sqrt{-k}) / 2$ as a product of Gauss sums. The Gross-Koblitz formula [8], [2, (11.2.12)] expresses these Gauss sums in terms of $p$-adic gamma functions. Specifically, it shows that there is an embedding of $\mathbb{Q}(\sqrt{-k})$ into the field $\mathbb{Q}_{p}$ of $p$-adic rationals which maps $(C+D \sqrt{-k}) / 2$ to a product of $p$-adic gamma functions, viz.,

$$
(C+D \sqrt{-k}) / 2=\prod_{u \in U} \Gamma_{p}(u / k) \quad\left(\text { in } \mathbb{Q}_{p}\right) .
$$

Here $\Gamma_{p}(z)$ is the $p$-adic gamma function, defined as in $[2,(9.3 .3)]$ to be the limit of

$$
(-1)^{N} \prod_{\substack{0<j<N \\ p \nmid j}} j
$$

as the positive integer $N$-adically approaches the $p$-adic integer $z$. By (1.1) and (2.8),

$$
(C-D \sqrt{-k}) / 2=p^{h} \prod_{u \in U} \Gamma_{p}(u / k)^{-1} .
$$

Adding (2.8) and (2.9), we obtain

$$
C=p^{h} \prod_{u \in U} \Gamma_{p}(u / k)^{-1}+\prod_{u \in U} \Gamma_{p}(u / k) .
$$


By (2.10) and (1.5),

$$
\prod_{u \in U} \Gamma_{p}(u / k) \equiv \prod_{u \in U}[p u / k] !^{-1}(\bmod p),
$$

so that (2.10) becomes

$$
C \equiv p^{h} \prod_{u \in U}[p u / k] !+\prod_{u \in U} \Gamma_{p}(u / k)\left(\bmod p^{2}\right) .
$$

It remains to show that

$$
\prod_{u \in U} \Gamma_{p}(u / k) \equiv(-(p-1) !)^{R} \prod_{u \in U}[p u / k] !^{-1}\left(1+\sum_{u \in U} u B_{u}\right)\left(\bmod p^{2}\right),
$$

which is equivalent, by Lemma 2.1 , to

$$
\begin{aligned}
\prod_{u \in U} \Gamma_{p}(u / k)^{-1} & \\
& \equiv(-(p-1) !)^{-R} \prod_{u \in U}[p u / k] !\left(1-\sum_{u \in U} u B_{u}\right)\left(\bmod p^{2}\right) .
\end{aligned}
$$

Define

$$
c=\max (r, 2) .
$$

Note that $k \mid\left(p^{c}-1\right)$. By $[2,(9.3 .8)]$,

$$
\Gamma_{p}(1-u / k) \equiv \Gamma_{p}\left(1+\left(p^{c}-1\right) u / k\right)\left(\bmod p^{2}\right) .
$$

Since $1+\left(p^{c}-1\right) u / k$ is a positive integer,

$$
\Gamma_{p}\left(1+\left(p^{c}-1\right) u / k\right)= \pm \prod_{\substack{1 \leq j \leq\left(p^{c}-1\right) u / k \\ p \nmid j}} j,
$$

by definition of $\Gamma_{p}$.

For $x \in \mathbb{R}$, define

$$
L(x)=x-k[x / k] .
$$

Since $p L(u / p)=k[L(u / p) p / k]+u$, we have

$$
u\left(p^{c}-1\right)=k[L(u / p) p / k]+p^{c} u-p L(u / p),
$$

which yields

$$
\left(p^{c}-1\right) u / k=[L(u / p) p / k]+p\left(u p^{c-1}-L(u / p)\right) / k .
$$

For each nonnegative integer $x$,

$$
(x p+1) \ldots(x p+p-1) \equiv(p-1) !\left(\bmod p^{2}\right),
$$

so it follows from (2.17) that 
$(2.18)$

$$
\begin{aligned}
\prod_{\substack{1 \leq j \leq\left(p^{c}-1\right) u / k \\
p \nmid j}} j \equiv(p-1) !^{\left(u p^{c-1}-L(u / p)\right) / k} & \\
& \times \prod_{j=1}^{[L(u / p) p / k]}\left(j+\left(u p^{c}-p L(u / p)\right) / k\right)\left(\bmod p^{2}\right) .
\end{aligned}
$$

The rightmost product on $j$ in (2.18) is congruent to

$$
\begin{aligned}
{[L(u / p) p / k] !(1} & \left.+\frac{u p^{c}-p L(u / p)}{k} \sum_{j=1}^{[L(u / p) p / k]} \frac{1}{j}\right) \\
\equiv[L(u / p) p / k] !\left(1-\frac{p L(u / p)}{k}\right. & \left.\sum_{j=1}^{[L(u / p) p / k]} \frac{1}{j}\right)\left(\bmod p^{2}\right) .
\end{aligned}
$$

Taking the product over all $u \in U$ in (2.15), (2.16), and (2.18), we thus obtain

$$
\begin{aligned}
& \pm \prod_{u \in U} \Gamma_{p}(1-u / k) \\
& \quad \equiv(p-1) !^{R\left(p^{c-1}-1\right)} \prod_{u \in U}[p u / k] !\left(1-\sum_{u \in U} u B_{u}\right)\left(\bmod p^{2}\right) .
\end{aligned}
$$

By the reflection formula for $\Gamma_{p}[2,(9.3 .5)]$, the left member of (2.19) equals $\pm \prod_{u \in U} \Gamma_{p}(u / k)^{-1}$. Thus (2.19) becomes

$$
\begin{aligned}
\pm \prod_{u \in U} & \Gamma_{p}(u / k)^{-1} \\
& \equiv(p-1) !^{R\left(p^{c-1}-1\right)} \prod_{u \in U}[p u / k] !\left(1-\sum_{u \in U} u B_{u}\right)\left(\bmod p^{2}\right) .
\end{aligned}
$$

The ambiguous sign on the left of (2.20) must be + by (2.11). Finally, the power of $(p-1)$ ! in $(2.20)$ can be simplified to $(-(p-1) !)^{-R}$, since $(p-1) !^{p} \equiv-1\left(\bmod p^{2}\right)$. This completes the proof of $(2.13)$.

The following corollary gives a congruence $\left(\bmod p^{2}\right)$ for Eisenstein's binomial coefficient $\left(\begin{array}{c}{[3 p / 7]} \\ {[p / 7]}\end{array}\right)$ in the case $k=7$.

Corollary 2.3. Let $k=7$ and let $p$ be an odd prime with $\left(\frac{-7}{p}\right)=1$, i.e., with $p \equiv 1,2$, or $4(\bmod 7)$. Then

$$
\begin{aligned}
Z & :=\left(\begin{array}{c}
{[3 p / 7]} \\
{[p / 7]}
\end{array}\right) \\
& \equiv(-1)^{[3 p / 7]}\left(p / C+C\left(B_{1}+2 B_{2}-3 B_{3}-1\right)\right)\left(\bmod p^{2}\right) .
\end{aligned}
$$

Proof. For brevity, write $p_{i}=[i p / 7], i=1,2,3,4$. Since $p \equiv 1,2$, or 4 modulo 7 , it follows that $Z=p_{3} ! /\left(p_{1} ! p_{2} !\right)$. For $k=7$, we have $h=1, U=$ 
$\{1,2,4\}$, and $R=1$. By $(2.7)$,

$$
C \equiv p_{1} ! p_{2} ! p_{4} ! p-\frac{(p-1) !\left(1+B_{1}+2 B_{2}+4 B_{4}\right)}{p_{1} ! p_{2} ! p_{4} !}\left(\bmod p^{2}\right) \text {. }
$$

Since $p_{3}+p_{4}=p-1$, we have

$$
p_{3} ! p_{4} ! \equiv-(-1)^{p_{3}}(\bmod p) .
$$

By (2.22) and (2.23),

$$
C \equiv-(-1)^{p_{3}} p / Z-\frac{(p-1) ! Z\left(1+B_{1}+2 B_{2}+4 B_{4}\right)}{p_{3} ! p_{4} !}\left(\bmod p^{2}\right) .
$$

By (2.23) and (2.24),

$$
C \equiv-Z(-1)^{p_{3}}(\bmod p) .
$$

Combining (2.24) and (2.25), we obtain

$$
C \equiv p / C-\frac{(p-1) ! Z\left(1+B_{1}+2 B_{2}+4 B_{4}\right)}{p_{3} ! p_{4} !}\left(\bmod p^{2}\right) .
$$

Solving (2.26) for $Z$ and using the equality $B_{4}=B_{3}$, we obtain

$$
Z \equiv(p / C-C)\left(1-B_{1}-2 B_{2}-4 B_{3}\right) Y\left(\bmod p^{2}\right),
$$

where

$$
Y=\frac{p_{3} ! p_{4} !}{(p-1) !}
$$

We proceed to compute $Y\left(\bmod p^{2}\right)$. Since

$$
\begin{aligned}
(p-1) ! & =(p-1) \ldots\left(p-p_{3}\right) \cdot p_{4} ! \equiv(-1)^{p_{3}} p_{3} ! p_{4} !\left(1-p \sum_{j=1}^{p_{3}} 1 / j\right) \\
& \equiv(-1)^{p_{3}} p_{3} ! p_{4} !\left(1-7 B_{3}\right)\left(\bmod p^{2}\right)
\end{aligned}
$$

we see that

$$
Y \equiv(-1)^{p_{3}}\left(1+7 B_{3}\right)\left(\bmod p^{2}\right) .
$$

By (2.27) and (2.29),

$$
Z \equiv(p / C-C)(-1)^{p_{3}}\left(1+3 B_{3}-B_{1}-2 B_{2}\right)\left(\bmod p^{2}\right),
$$

which proves $(2.21)$.

ExAmPle 2.4. Let $k=7$ and $p=37$. Then $R=h=1, p_{1}=5, p_{2}=10$, $p_{3}=15, p_{4}=21,-B_{2} \equiv B_{1} \equiv 74\left(\bmod 37^{2}\right), B_{3}=B_{4} \equiv 814\left(\bmod 37^{2}\right)$, and

$$
4 p=148=C^{2}+7 D^{2} \quad \text { with } \quad C= \pm 6, D= \pm 4 .
$$

Congruence (1.2) becomes

$$
C \equiv 2(-37)^{-1} \equiv-1(\bmod 7)
$$


and congruence (2.7) becomes

$$
C \equiv 37 p_{1} ! p_{2} ! p_{4} !-\frac{36 !\left(1+B_{1}+2 B_{2}+4 B_{4}\right)}{p_{1} ! p_{2} ! p_{4} !} \equiv 6\left(\bmod 37^{2}\right) .
$$

Both of these congruences must hold for the same choice of $C= \pm 6$, which in this case is $C=6$. With $C=6$, congruence (2.21) becomes

$$
Z \equiv-37 / 6-6\left(B_{1}+2 B_{2}-3 B_{3}-1\right) \equiv 265\left(\bmod 37^{2}\right) .
$$

To verify this, note that

$$
Z=\left(\begin{array}{c}
15 \\
5
\end{array}\right)=3003=2\left(37^{2}\right)+265 .
$$

3. Determination of $C\left(\bmod p^{2}\right)$ when $p=2$. We begin by discussing properties of the 2-adic gamma function $\Gamma_{2}$, which can be defined just as we defined $\Gamma_{p}$ below (2.8), with $p$ replaced by 2 . (Although $\Gamma_{p}$ is defined in $[2,(9.3 .3)]$ for $p>2$ only, this definition is also valid for $p=2$, because the congruence $[2,(9.3 .2)]$ holds for $p=2$ whenever the modulus exceeds 4.)

Given a 2-adic integer

$$
z=z_{0}+z_{1} 2+z_{2} 4+z_{3} 8+\ldots, \quad z_{i} \in\{0,1\},
$$

we have the reflection formula

$$
\Gamma_{2}(z) \Gamma_{2}(1-z)=(-1)^{M(z)},
$$

where

$$
M(z)=z_{1}+1 .
$$

(The reflection formula $[2,(9.3 .5)]$ for $\Gamma_{p}$, which was used below (2.19), does not reduce to (3.1) when $p=2$; it holds only for $p>2$.) To verify (3.1), note that for integers $n, N$ with $n>2$ and $0<N<2^{n}$,

$$
\prod_{\substack{0<j<N \\ j \text { odd }}} j \prod_{\substack{0<j<2^{n}+1-N \\ j \text { odd }}} j \equiv(-1)^{M(N)}\left(\bmod 2^{n}\right),
$$

and when $N$ approaches $z$ (2-adically) in (3.2), we obtain (3.1) by the continuity of $\Gamma_{2}(z)$ and $M(z)$.

We shall need the Gross-Koblitz formula [2, (11.2.12)] for $p=2$. This formula was originally presented [8] for $p>2$, but it has been proved for $p=2$ as well (see Coleman [4], [5]).

Theorem 3.1. Suppose that $p=2$ and $\left(\frac{-k}{p}\right)=1$, so that $k \equiv 7(\bmod 8)$ (with $k$ squarefree). Let $H$ denote the class number of $\mathbb{Q}(\sqrt{-8 k})$. Then

$$
C \equiv 2^{h}+(-1)^{R+H / 4}(\bmod 4) \text {. }
$$

In particular, $C \equiv-1(\bmod 4)$ when $k=7$, while for $k>7$,

$$
C \equiv(-1)^{R+H / 4}(\bmod 4) \text {. }
$$


Proof. We need only prove (3.3), since (3.4) follows from (3.3) and the fact that $h>1$ when $k>7, k \equiv 7(\bmod 8)$.

In light of the proof of Theorem 2.2 and the remarks preceding Theorem 3.1, we see that (2.12) is valid for $p=2$ as well as for $p>2$. Thus,

$$
C \equiv 2^{h}+\prod_{u \in U} \Gamma_{2}(u / k)(\bmod 4) .
$$

It remains to prove that

$$
\prod_{u \in U} \Gamma_{2}(u / k) \equiv(-1)^{R+H / 4}(\bmod 4) .
$$

By $(3.1)$,

$$
\prod_{u \in U} \Gamma_{2}(u / k) \Gamma_{2}(1-u / k)=(-1)^{\sum_{u \in U} M(u / k)} .
$$

Since $k \equiv 7(\bmod 8)$, it is easily checked that $M(u / k)$ is odd if and only if $u \equiv 0$ or $3(\bmod 4)$. Thus

$$
\sum_{u \in U} M(u / k) \equiv N(0,3,4,7)(\bmod 2),
$$

where $N(a, b, c, d)$ denotes the number of elements in $U$ congruent to one of $a, b, c, d$ modulo 8. Combining (3.5)-(3.7), we see that it remains to prove

$$
\prod_{u \in U} \Gamma_{2}(1-u / k) \equiv(-1)^{R+H / 4+N(0,3,4,7)}(\bmod 4) .
$$

Since the congruence $[2,(9.3 .8)]$ holds for $p=2$ (as well as for $p>2$ ) when the modulus exceeds 4 , it can be proved, analogous to the proof of (2.15)-(2.16), that

$$
\Gamma_{2}(1-u / k) \equiv \Gamma_{2}\left(1+\left(2^{r}-1\right) u / k\right)=(-1)^{u+1} \prod_{\substack{1 \leq j \leq\left(2^{r}-1\right) u / k \\ j \text { odd }}} j(\bmod 4) .
$$

It follows that $\Gamma_{2}(1-u / k) \equiv \pm 1(\bmod 4)$, with $\Gamma_{2}(1-u / k) \equiv-1(\bmod 4)$ if and only if $\left(2^{r}-1\right) u / k \equiv 3,4,5$, or $6(\bmod 8)$. Thus $\Gamma_{2}(1-u / k) \equiv-1$ $(\bmod 4)$ if and only if $u \equiv 3,4,5$, or $6(\bmod 8)$, and so

$$
\begin{aligned}
\prod_{u \in U} \Gamma_{2}(1-u / k) & \equiv(-1)^{\sum_{u \in U} u+\sum_{u \in U} 1+N(3,4,5,6)} \\
& =(-1)^{R+N(7,0,1,2)}(\bmod 4) .
\end{aligned}
$$

By (3.8) and (3.10), it remains to prove that

$$
\sum_{\substack{u \in U \\ u \equiv 1,2,3,4(\bmod 8)}} 1 \equiv H / 4(\bmod 2) .
$$


We have

$$
2 \sum_{\substack{u \in U \\ u \equiv 1,2,3,4(\bmod 8)}} 1=A+B
$$

where

$$
A=\sum_{\substack{j=1 \\ j, k=1 \\ j \equiv 1,3,4(\bmod 8)}}^{k-1} 1, \quad B=\sum_{\substack{j=1 \\ j \equiv 1,2,3,4(\bmod 8)}}^{k-1}\left(\frac{j}{k}\right) .
$$

We proceed to prove that 4 divides $A$. For the Möbius function $\mu$,

$$
A=\sum_{\substack{j=1 \\ j \equiv 1,2,3,4(\bmod 8)}}^{k-1} \sum_{\substack{d|k \\ d| j}} \mu(d)=\sum_{d \mid k} \mu(d) \sum_{\substack{n=1 \\ n \equiv d, 2 d, 3 d, 4 d(\bmod 8)}}^{k / d-1} 1,
$$

where we have written $j=d n$. The inner sum on $n$ in (3.14) is congruent to $0,3,1$, or $0(\bmod 4)$ according as $d \equiv 1,3,5$, or $7(\bmod 8)$. Thus,

$$
A \equiv \sum_{\substack{d \mid k \\ d \equiv 5(\bmod 8)}} \mu(d)-\sum_{\substack{d \mid k \\ d \equiv 3(\bmod 8)}} \mu(d)(\bmod 4) .
$$

Write $k=p_{1} \ldots p_{\nu}$, where the $p_{i}$ are distinct primes. By (3.15),

$$
A \equiv \sum_{\substack{d \mid k \\ d \equiv 5(\bmod 8)}} \mu(d)\left(1-(-1)^{\nu}\right)(\bmod 4) .
$$

If $\nu$ is even, then (3.16) shows that $4 \mid A$, so suppose that $\nu$ is odd. If $\nu=1$, then $4 \mid A$ because there are no terms in the sum in (3.16) (since $k \equiv 7$ $(\bmod 8))$. Thus suppose that $\nu$ is odd $\geq 3$. By $(3.16)$,

$$
\begin{aligned}
A & \equiv 2 \sum_{\substack{d \mid k \\
d \equiv 5(\bmod 8)}} \mu(d) \equiv 2 \sum_{\substack{d \mid k \\
d \equiv 5(\bmod 8)}} 1 \\
& =\frac{1}{2} \sum_{d \mid k} \sum_{\chi(\bmod 8)} \chi(5 d)=\frac{1}{2} \sum_{\chi(\bmod 8)} \chi(5) \sum_{d \mid k} \chi(d) \\
& =\frac{1}{2} \sum_{\chi(\bmod 8)} \chi(5) \prod_{i=1}^{\nu}\left(1+\chi\left(p_{i}\right)\right)(\bmod 4) .
\end{aligned}
$$

Each product on $i$ above is divisible by 8 , since

$$
\prod_{i=1}^{\nu}\left(1+\chi\left(p_{i}\right)\right)=0 \text { or } 2^{\nu}
$$

By (3.17), this completes the proof that $4 \mid A$. 
In view of $(3.11)-(3.12)$, it now suffices to prove that

$$
B=H / 2 \text {. }
$$

Since

$$
\sum_{\substack{j=1 \\ j \equiv 3,4(\bmod 8)}}^{k-1}\left(\frac{j}{k}\right)=\sum_{\substack{j=1 \\ j \equiv 3(\bmod 8)}}^{k-1}\left\{\left(\frac{j}{k}\right)+\left(\frac{k-j}{k}\right)\right\}=0,
$$

$$
B=\sum_{\substack{j=1 \\ j \equiv 1,2(\bmod 8)}}^{k-1}\left(\frac{j}{k}\right) .
$$

Write $k=8 f+7$. Since $\left(\frac{2}{k}\right)=1,(3.19)$ yields

$$
\begin{aligned}
B & =\sum_{m=0}^{f}\left(\frac{8 m+1}{k}\right)+\sum_{m=0}^{f}\left(\frac{8 m+2}{k}\right) \\
& =\sum_{m=0}^{f}\left(\frac{m+f+1}{k}\right)+\sum_{m=0}^{f}\left(\frac{m+2 f+2}{k}\right) \\
& =\sum_{m=f+1}^{3 f+2}\left(\frac{m}{k}\right)=\sum_{k / 8<j<3 k / 8}\left(\frac{j}{k}\right)=2 \sum_{k / 4<j<3 k / 8}\left(\frac{j}{k}\right),
\end{aligned}
$$

where the last equality follows from the last statement in Berndt [1, Cor. 7.2, p. 281]. On the other hand, by [1, (7.6), p. 282],

$$
\sum_{k / 4<j<3 k / 8}\left(\frac{j}{k}\right)=H / 4 \text {. }
$$

Combining (3.20) and (3.21), we obtain (3.18).

EXAMPle 3.2. Let $k=143$ and $p=2$. Then $h=10, R=\phi(k) / 4-h / 2$ $=115, H=12$, and

$$
4 p^{h}=4096=C^{2}+143 D^{2} \quad \text { with } \quad C= \pm 53, D= \pm 3 .
$$

By (1.3) with $w=11, t=13$, and $b=5$,

$$
C \equiv 2^{6}(-1)^{115+120 / 20}=-64(\bmod 13) .
$$

By (3.4)

$$
C \equiv(-1)^{115+12 / 4}=1(\bmod 4) .
$$

Both of these congruences must hold for the same choice of $C= \pm 53$, which in this case is $C=53$.

The following theorem expresses the results of Theorem 3.1 in a form independent of class numbers. 
Theorem 3.3. Suppose that $p=2$ and $\left(\frac{-k}{p}\right)=1$, so that $k \equiv 7(\bmod 8)$ (with $k$ squarefree). Then $C \equiv-1(\bmod 4)$, if $k=7 ; C \equiv \delta(\bmod 4)$, if $k$ is a prime $>7$, where $\delta= \pm 1$ is defined by

$$
\delta \equiv((k-1) / 2) !(-1)^{(k+1) / 8}(\bmod k) ;
$$

$C \equiv\left(\frac{2 Q}{P}\right)(\bmod 4)$, if $k=P Q$ for primes $P, Q$; and $C \equiv 1(\bmod 4)$, if $k$ has more than two prime factors.

Proof. Theorem 3.3 can be deduced from (3.3) by applying known congruences for class numbers modulo powers of 2 . Such congruences are discussed in the second chapter of the book of Urbanowicz and Williams [11]. Specifically, for prime $k>7$, one uses a theorem of Mordell [9], [11, Theorem 8 , p. 52] together with a result of Berndt [1, Corollary 7.4], [11, p. 59]. For $k=P Q$, one uses the results in [11, p. 60] and [11, Theorem 13, p. 62]. Finally, when $k$ has more than two prime factors, one uses the facts that $H / 4$ and $R=\phi(k) / 4-h / 2$ are both even; this is a consequence of Gauss's theory of genera $[11,(1)$, p. 51$]$.

\section{Determination of $C\left(\bmod p^{2}\right)$ when $k=8$}

THEOREM 4.1. Let $k=8$ and let $p$ be a prime with $\left(\frac{-8}{p}\right)=1$, i.e., with $p \equiv 1$ or $3(\bmod 8)$. Then there are integers $C, D$ (with $C$ unique) such that (1.10) and (1.11) hold.

Proof. This is proved for $p \equiv 1(\bmod 8)$ in $[2$, Theorem 9.4.5], so assume that $p \equiv 3(\bmod 8)$. In $[2$, Theorem 12.9 .6$]$, it is shown that there are integers $C, D$ satisfying (1.10) such that $(C+D \sqrt{-8}) / 2$ equals a certain octic Eisenstein sum. This Eisenstein sum can in turn be expressed as a quotient of an octic Gauss sum over a quadratic Gauss sum, by [2, Theorem 12.1.1]. Using the Gross-Koblitz formula [2, (11.2.12)] and the fact that

$$
(q-1) / 8=(3 p-1) / 8+p(p-3) / 8,
$$

we find that the analog of $(2.8)$ is

$$
(C+D \sqrt{-8}) / 2=\Gamma_{p}\left(\frac{1}{8}\right) \Gamma_{p}\left(\frac{3}{8}\right) \Gamma_{p}\left(\frac{1}{2}\right)^{-1} \quad\left(\text { in } \mathbb{Q}_{p}\right) .
$$

Therefore,

$$
(C-D \sqrt{-8}) / 2=p \Gamma_{p}\left(\frac{1}{8}\right)^{-1} \Gamma_{p}\left(\frac{3}{8}\right)^{-1} \Gamma_{p}\left(\frac{1}{2}\right)
$$

and addition of these equalities yields 


$$
\pm C=p \Gamma_{p}\left(\frac{1}{8}\right)^{-1} \Gamma_{p}\left(\frac{3}{8}\right)^{-1}+\Gamma_{p}\left(\frac{1}{8}\right) \Gamma_{p}\left(\frac{3}{8}\right),
$$

since $\Gamma_{p}\left(\frac{1}{2}\right)= \pm 1$ by the reflection formula. The proof of (2.20) works for $k=8$, and shows that the analog of $(2.20)$ is

$$
\begin{aligned}
\pm \Gamma_{p}\left(\frac{1}{8}\right)^{-1} & \Gamma_{p}\left(\frac{3}{8}\right)^{-1} \\
& \equiv(p-1) !^{(p-1) / 2}\left[\frac{p}{8}\right] !\left[\frac{3 p}{8}\right] !\left(1-B_{1}-3 B_{3}\right)\left(\bmod p^{2}\right) .
\end{aligned}
$$

Equivalently,

$$
\begin{aligned}
& \pm \Gamma_{p}\left(\frac{1}{8}\right) \Gamma_{p}\left(\frac{3}{8}\right) \\
& \quad \equiv(p-1) !^{(1-p) / 2}\left[\frac{p}{8}\right] !^{-1}\left[\frac{3 p}{8}\right] !^{-1}\left(1+B_{1}+3 B_{3}\right)\left(\bmod p^{2}\right) .
\end{aligned}
$$

Write $\tau=(p-1) / 2$. We proceed to examine the factor $(p-1) !^{-\tau}$ in (4.4). Since $\tau !^{2} \equiv 1(\bmod p)$,

$$
\begin{aligned}
(p-1) ! & =(p-1) \ldots(p-\tau) \cdot \tau ! \\
& \equiv-\tau !^{2}+p \sum_{j=1}^{\tau} 1 / j \equiv-\tau !^{2}+8 B_{4}\left(\bmod p^{2}\right) .
\end{aligned}
$$

Write

$$
\tau !=\varepsilon+x p, \quad \text { where } \quad x \in \mathbb{Z}, \varepsilon= \pm 1 .
$$

Then $\tau !^{2} \equiv 1+2 \varepsilon x p\left(\bmod p^{2}\right)$, so that by $(4.5)$,

$$
(p-1) ! \equiv-1-2 \varepsilon x p+8 B_{4}\left(\bmod p^{2}\right) .
$$

Since $\tau$ is odd, the binomial theorem and (4.7) yield

$$
(p-1) !^{\tau} \equiv-1-4 B_{4}+\operatorname{\varepsilon xp}\left(\bmod p^{2}\right),
$$

so by (4.6),

$$
(p-1) !^{-\tau} \equiv-1+4 B_{4}-\varepsilon x p \equiv 4 B_{4}-\varepsilon \tau !\left(\bmod p^{2}\right) .
$$

By (4.8) and (4.6),

$$
(p-1) !^{-\tau} / \tau ! \equiv \varepsilon\left(4 B_{4}-1\right)\left(\bmod p^{2}\right) .
$$

Therefore (4.4) becomes

$$
\begin{aligned}
\pm \Gamma_{p}\left(\frac{1}{8}\right) \Gamma_{p}\left(\frac{3}{8}\right) & \equiv\left(\begin{array}{l}
{[p / 2]} \\
{[p / 8]}
\end{array}\right)\left(1-4 B_{4}\right)\left(1+B_{1}+3 B_{3}\right) \\
& \equiv\left(\begin{array}{l}
{[p / 2]} \\
{[p / 8]}
\end{array}\right)\left(1+B_{1}+3 B_{3}-4 B_{4}\right)\left(\bmod p^{2}\right) .
\end{aligned}
$$


Consequently, (4.2) becomes

$$
\pm C \equiv p\left(\begin{array}{l}
{[p / 2]} \\
{[p / 8]}
\end{array}\right)^{-1}+\left(\begin{array}{l}
{[p / 2]} \\
{[p / 8]}
\end{array}\right)\left(1+B_{1}+3 B_{3}-4 B_{4}\right)\left(\bmod p^{2}\right) .
$$

The congruence for $C(\bmod p)$ in $[2$, Theorem 12.9.7] shows that the + sign in (4.11) is correct. To complete the proof of (1.11), it remains to show that

$$
1+B_{1}+3 B_{3}-4 B_{4} \equiv 2-2^{p-1}-y / 8\left(\bmod p^{2}\right),
$$

where $y$ is the integer defined by (1.12). The proof of (4.12) proceeds just as the analogous proof in [2, Theorem 9.4.5] (with $q=p^{2}$ in place of $p$ ).

The following corollary gives a congruence $\left(\bmod p^{2}\right)$ for Eisenstein's binomial coefficient $\left(\begin{array}{c}{[p / 2]} \\ {[p / 8]}\end{array}\right)$ when $k=8$.

Corollary 4.2. Let $k=8$, and let $p$ be a prime with $\left(\frac{-8}{p}\right)=1$, i.e., with $p \equiv 1$ or $3(\bmod 8)$. Then

$$
W:=\left(\begin{array}{l}
{[p / 2]} \\
{[p / 8]}
\end{array}\right) \equiv(C-p / C)\left(2^{p-1}+y / 8\right)\left(\bmod p^{2}\right),
$$

where $y$ is defined in (1.12).

Proof. By (1.11),

$$
C \equiv p / W+W\left(2-2^{p-1}-y / 8\right)\left(\bmod p^{2}\right) .
$$

In particular, since $p$ divides $y$ by (4.12),

$$
C \equiv W(\bmod p) .
$$

By (4.14) and (4.15),

$$
C \equiv p / C+W\left(2-2^{p-1}-y / 8\right)\left(\bmod p^{2}\right) .
$$

Solving (4.16) for $W$ yields

$$
W \equiv(C-p / C)\left(2-2^{p-1}-y / 8\right)^{-1}\left(\bmod p^{2}\right),
$$

and (4.13) follows.

EXAMPLE 4.3. Let $k=8$ and $p=43$. Then $h=1, y \equiv 1462\left(\bmod 43^{2}\right)$, $\left(\begin{array}{c}{[p / 2]} \\ {[p / 8]}\end{array}\right)=\left(\begin{array}{c}21 \\ 5\end{array}\right)=20349$, and

$$
4 p=172=C^{2}+8 D^{2} \quad \text { with } \quad C= \pm 10, D= \pm 3 .
$$

The congruence in (1.10) becomes

$$
C \equiv 2(-1)^{5+21}=2(\bmod 8)
$$

and the congruence (1.11) becomes

$$
C \equiv \frac{43}{20349}+20349\left(2-2^{42}-\frac{1462}{8}\right) \equiv 10\left(\bmod 43^{2}\right) .
$$


Both of these congruences must hold for the same choice of $C= \pm 10$, which in this case is $C=10$. With $C=10,(4.13)$ becomes

$$
W \equiv\left(10-\frac{43}{10}\right)\left(2^{42}+\frac{1462}{8}\right) \equiv 10\left(\bmod 43^{2}\right) .
$$

To verify this, observe that

$$
W=20349=11\left(43^{2}\right)+10 .
$$

\section{References}

[1] B. C. Berndt, Classical theorems on quadratic residues, Enseign. Math. 22 (1976), 261-304.

[2] B. C. Berndt, R. J. Evans and K. S. Williams, Gauss and Jacobi Sums, Wiley, 1998.

[3] S. Chowla, B. Dwork and R. J. Evans, On the mod $p^{2}$ determination of $\left(\begin{array}{l}(p-1) / 2 \\ (p-1) / 4\end{array}\right)$, J. Number Theory 24 (1986), 188-196.

[4] R. F. Coleman, The Gross-Koblitz formula, in: Galois Representations and Arithmetic Algebraic Geometry, Y. Ihara (ed.), Adv. Stud. Pure Math. 12, NorthHolland, Amsterdam, 1987, 21-52.

[5] —, p-adic Analysis, Lecture Notes in Math. 1454, Springer, Berlin, 1990, p. 193 (Corrigenda to reference \#4).

[6] R. Crandall, K. Dilcher and C. Pomerance, A search for Wieferich and Wilson primes, Math. Comp. 66 (1997), 433-449.

[7] R. J. Evans, Classical congruences for parameters in binary quadratic forms, Finite Fields Appl. 7 (2001), 110-124.

[8] B. Gross and N. Koblitz, Gauss sums and the p-adic $\Gamma$-function, Ann. of Math. (2) 109 (1979), 569-581.

[9] L. J. Mordell, The congruence $((p-1) / 2) ! \equiv \pm 1(\bmod p)$, Amer. Math. Monthly 68 (1961), 145-146.

[10] L. Stickelberger, Über eine Verallgemeinerung der Kreistheilung, Math. Ann. 37 (1890), 321-367.

[11] J. Urbanowicz and K. S. Williams, Congruences for L-functions, Math. Appl. 511, Kluwer, Dordrecht, 2000.

Department of Mathematics

University of California at San Diego

La Jolla, CA 92093-0112, U.S.A.

E-mail: revans@ucsd.edu 